\title{
The observable signature of late heating of the Universe during cosmic reionization
}

\author{
Anastasia Fialkov, ${ }^{1,2}$ Rennan Barkana, ${ }^{1}$ Eli Visbal ${ }^{3,4,5}$ \\ ${ }^{1}$ Raymond and Beverly Sackler School of Physics and Astronomy, \\ Tel Aviv University, Tel Aviv 69978, Israel \\ ${ }^{2}$ Département de Physique, Ecole Normale Supérieure, \\ CNRS, 24 rue Lhomond, 75005 Paris, France \\ ${ }^{3}$ Department of Astronomy, Columbia University, \\ 550 West 120th Street, New York, NY 10027, USA \\ ${ }^{4}$ Jefferson Laboratory of Physics, Harvard University, \\ Cambridge, MA 02138, USA \\ ${ }^{5}$ Institute for Theory \& Computation, Harvard University, \\ 60 Garden Street, Cambridge, MA 02138, USA
}

February 6, 2014

\begin{abstract}
Models and simulations ${ }^{1,2,3,4}$ of the epoch of reionization predict that spectra of the 21-cm transition of atomic hydrogen will show a clear fluctuation peak, at a redshift and scale, respectively, that mark the central stage of reionization and the characteristic size of ionized bubbles. This is based on the assumption $\stackrel{5,6,7}{~ t h a t ~ t h e ~ c o s m i c ~ g a s ~ w a s ~ h e a t e d ~}$ by stellar remnants - particularly X-ray binaries - to temperatures well above the cosmic microwave background at that time $(\sim 30 \mathrm{~K})$. Here we show instead that the hard spectra (that is, spectra with more high-energy photons than low-energy photons) of X-ray binaries ${ }^{8.9}$ make such heating ineffective, resulting in a delayed and spatially uniform heating
\end{abstract}


that modifies the 21-cm signature of reionization. Rather than looking for a simple rise and fall of the large-scale fluctuations (peaking at several millikelvin), we must expect a more complex signal also featuring a distinct minimum (at less than a millikelvin) that marks the rise of the cosmic mean gas temperature above the microwave background. Observing this signal, possibly with radio telescopes in operation today, will demonstrate the presence of a cosmic background of hard X-rays at that early time.

While stellar remnants at high redshift have been previously considered, a more reliable prediction of the radiative feedback from X-ray binaries (XRBs) is now possible due to a recent detailed population synthesis simulation of their evolution across cosmic time ${ }^{8.9}$. This simulation was calibrated to all available observations in the local and low redshift Universe, and it predicts the evolution of the luminosity and X-ray spectrum of XRBs with redshift. In particular, high-mass XRBs (especially black hole binaries) should dominate, with a ratio at high redshift of bolometric X-ray luminosity to star-formation rate (SFR) of

$$
\frac{L_{X}}{\mathrm{SFR}}=3 \times 10^{40} f_{X} \mathrm{erg} \mathrm{s}^{-1} M_{\odot}^{-1} \mathrm{yr}
$$

We have allowed for an uncertainty in the X-ray efficiency with an extra parameter in eq. 1, where $f_{X}=1$ indicates our standard value. We focus on XRBs as the most natural heating source, since other observed sources should be sub-dominant at high redshift (see Methods section).

Previous calculations of X-ray heating ${ }^{6,10,11,12,13}$ have assumed power-law spectra that place most of the X-ray energy at the low-energy end, where the mean free path of the soft X-rays is relatively short. This means that most of the emitted X-rays are absorbed soon after they are emitted, before much energy is lost due to cosmological effects. The absorbed energy is then enough to heat the gas by the time of reionization to $\sim 10$ times the temperature of the Cosmic Microwave Background (CMB; see Methods section). Thus, it is generally assumed 
that reionization occurs when $T_{\text {gas }} \gg T_{\mathrm{CMB}}$, a limit referred to as saturated heating since the $21-\mathrm{cm}$ intensity then becomes independent of $T_{\text {gas }}$ and mainly dependent on ionization and density. A different possibility whereby heating is delayed until reionization has only been previously considered as a fringe case of having an unusually low X-ray luminosity to SFR ratio $\underline{10,11}$.

However, the average radiation from XRBs is expected to have a much harder spectrum (Fig. 1) whose energy content (per logarithmic frequency interval) peaks at $\sim 3 \mathrm{keV}$. Photons above a (roughly redshift-independent) critical energy of $\sim 1 \mathrm{keV}$ have such a long mean free path that by the start of reionization, most of these photons have not yet been absorbed, and the absorbed ones came from distant sources that were effectively dimmed due to cosmological redshift effects. This reduces the absorbed energy by about a factor of 5, enough to push the moment at which the mean gas temperature equals that of the CMB into the expected redshift range of cosmic reionization. This moment (termed the "heating transition") is a key milestone in 21-cm cosmology.

The immediate effect of the newly predicted late heating is to give the cosmic gas more time to cool adiabatically to well below the CMB temperature, thus producing mean $21-\mathrm{cm}$ absorption that reaches a maximum depth in the range -110 to $-180 \mathrm{mK}$ at $z \sim 15-19$ (Fig. 2). This may make it easier for experiments to detect the global 21-cm spectrum from before reionization and thus probe the corresponding early galaxies. Global experiments are most sensitive to the frequency derivative of the $21-\mathrm{cm}$ brightness temperature; late heating extends the steep portion of the spectrum to higher frequencies, moving the maximum positive derivative to a $\sim 10 \%$ higher frequency (where the foregrounds are significantly weaker) while also changing the value of this maximum derivative by $\pm 10 \%$.

We illustrate the possibilities for the timing of reionization with two example cases, one (our "early" reionization case) which has an optical depth to reionization equal to the most 


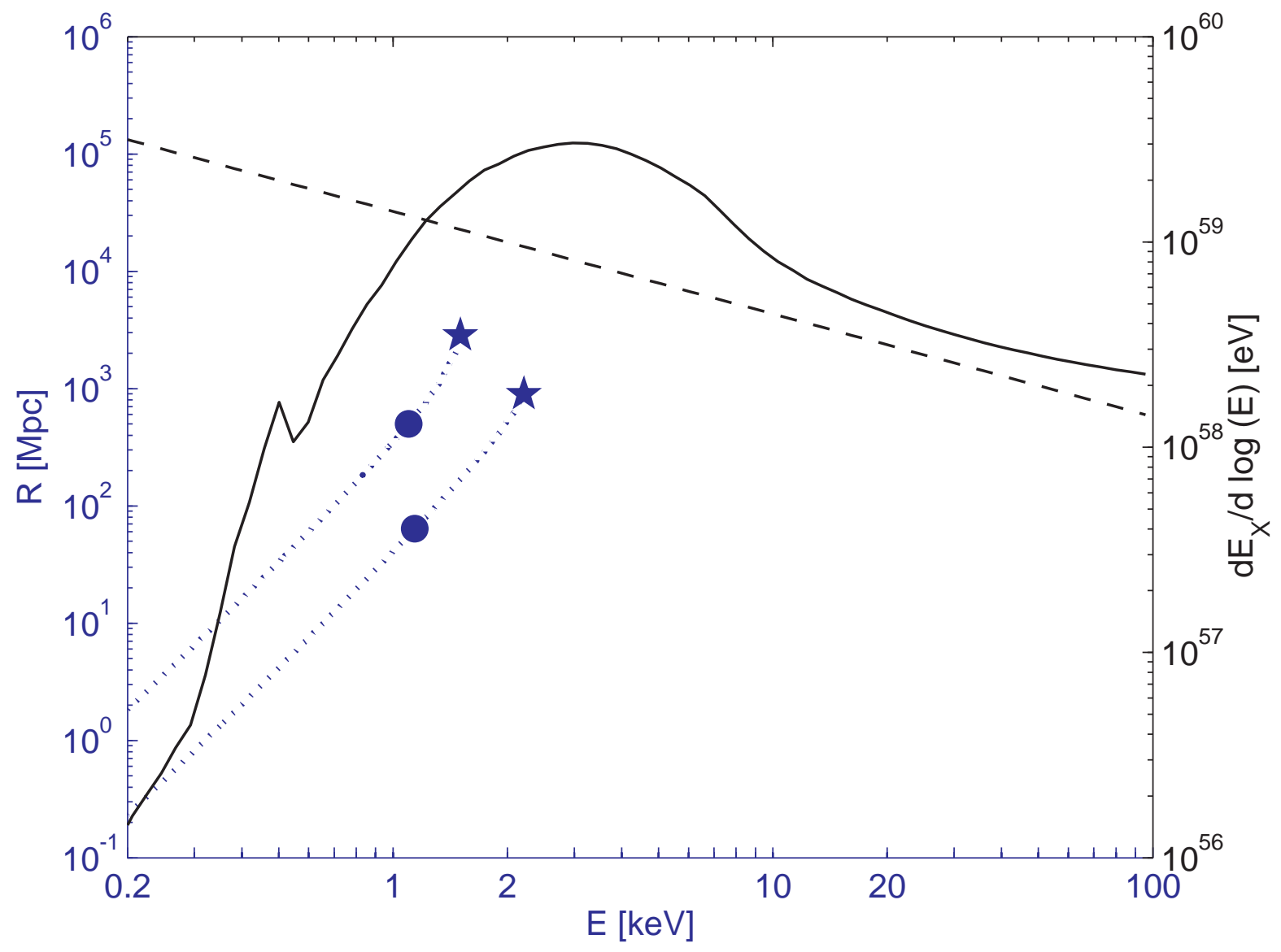

Figure 1: X-ray spectra, mean free paths, and horizons. We compare the expected spectrum of XRBs at high redshift (solid curve) from population synthesis models $\frac{8.9}{}$ to the soft power-law spectrum (dashed curve) adopted in previous studies $\frac{6.10 .11 .12 .13}{}$. Both indicate the distribution into X-ray photons with energy $E$ of the total X-ray energy $E_{X}$ produced per solar mass of newly-formed stars, for $f_{X}=1$ in eq. 11. The X-ray emission of XRBs should be dominated by the most massive systems in their high (that is, bright) state ${ }^{9}$, which is dominated by thermal disk emission, with little emission expected or seen ${ }^{8,27,28}$ below $\sim 1 \mathrm{keV}$. We also show the mean free paths (dotted curves) of X-ray photons arriving at $z=10$ (top) or $z=30$ (bottom). For each of these redshifts, we also indicate the effective horizon (defined as a 1/e drop-off, like a mean free path) from the combined effect of cosmological redshift and time retardation of sources $(\bullet)$, and the distance to $z=65(\star)$, the formation redshift of the first $\operatorname{star}^{29,30}$ (where we cut off the mean free path curves). Note the separate $y$ axes that indicate energy content for the spectra (right) or comoving distance for the other quantities (left). Note that "log" denotes a natural logarithm. 

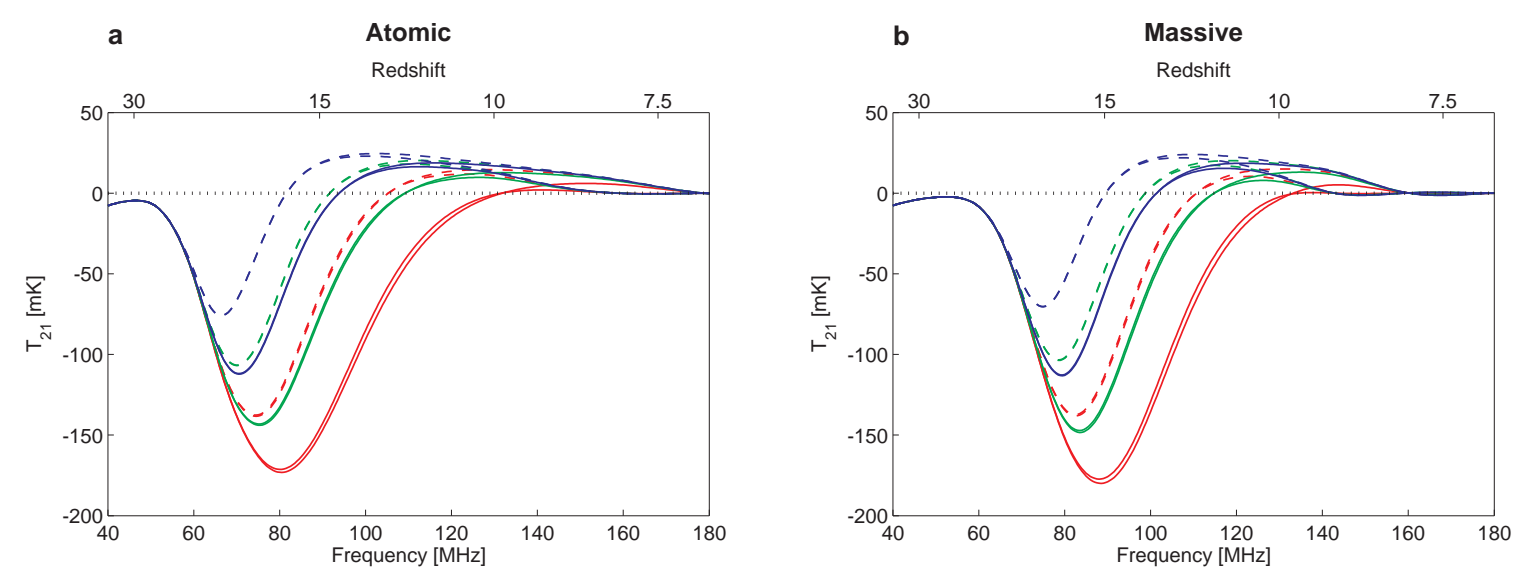

Figure 2: The global 21-cm spectrum. We show the cosmic mean 21-cm brightness temperature $\left(T_{21}\right)$ relative to the CMB versus observed frequency, for the new XRB spectrum ${ }^{9}$ (solid curves) and for the previously-adopted soft spectrum (dashed curves); note also the fiducial dotted line at $T_{21}=0$. We consider our standard $f_{X}=1$ case (green curves), as well as $f_{X}=1 / \sqrt{10}$ (red curves) or $\sqrt{10}$ (blue curves), each with either early or late reionization (The early case is always closer to $T_{21}=0$ ). We consider our two cases for galactic halos, atomic cooling (a) or massive halos (b), where the latter has a higher star-formation efficiency (see Methods section). With the old spectrum, the prediction with $f_{X}=1$ was for a deepest absorption signal of $T_{21}=-107$ to $-103 \mathrm{mK}$ at $z=17-19$ (ranges indicate our various halo and reionization cases), and a minimum mean $T_{\text {gas }}=8.4-10.4 \mathrm{~K}$ at $z=18.5-20$. With the new spectrum, these values change to $T_{21}=-148$ to $-143 \mathrm{mK}$ at $z=16-18$, and $T_{\text {gas }}=6.9-8.4 \mathrm{~K}$ at $z=16.5-18$. Since star formation in the models is normalized based on reionization, the massive halo case has less star formation and heating (compared to the atomic cooling case) prior to reionization, and it produces a sharper global $21-\mathrm{cm}$ signal. Note that these plots extend to higher redshift than is our main focus in this paper, so the high-redshift (i.e., low-frequency) drop to the trough could begin at somewhat lower frequencies than indicated if there is a significant contribution from lower-mass halos that we have not included here $\underline{26}$. 
likely value according to CMB experiments $\frac{14,15}{}$, and the other ("late" reionization) which is $1 \sigma$ below the central optical depth value and is more in line with possible hints of a late end to reionization ${ }^{16}$. In each case we also consider two possibilities for the dark matter halos that host galaxies. Since we focus here on reionization, by which time star formation in the small host halos of the first stars has been shut off by Lyman-Werner radiation ${ }^{17,18,19}$, we consider a minimum mass set by the need for efficient atomic cooling, or a minimum mass higher by an order of magnitude; the latter is an example of the case where lower-mass halos are inefficient at star formation, e.g., due to internal supernova feedback ${ }^{20,21}$. We refer to these two cases as "atomic cooling" and "massive halos", respectively.

The second key consequence of X-ray heating by a hard spectrum is a suppression of $21-\mathrm{cm}$ fluctuations due to heating. Under the previously assumed soft spectra, the short typical distance traveled by the X-ray photons was found to produce large fluctuations in the gas temperature and thus in the $21-\mathrm{cm}$ intensity around the time of the heating transition, regardless of when this transition occurred ${ }^{10,11,22}$. However, the larger source distances associated with a hard spectrum lead to a much more uniform heating, with correspondingly low temperature fluctuations even around the time of the heating transition, when the 21-cm intensity is most sensitive to the gas temperature. This trend is strengthened by late heating, as it occurs at a time when the heating sources are no longer as rare and strongly biased as they would be in the case of an earlier heating era. Thus, heating with a hard X-ray spectrum is predicted to produce a new signature in the 21-cm fluctuation signal: a deep minimum during reionization (Fig. 3; also shown versus redshift in Extended Data Fig. 1). This results from the low level of gas temperature fluctuations in combination with a suppression of the 21-cm impact of other types of fluctuations (i.e., in density and ionization); in particular, right at the heating transition, the cosmic mean $21-\mathrm{cm}$ intensity is (very nearly) zero, and thus all fluctuations other than those in gas temperature disappear (to linear order) from the 21-cm sky. 

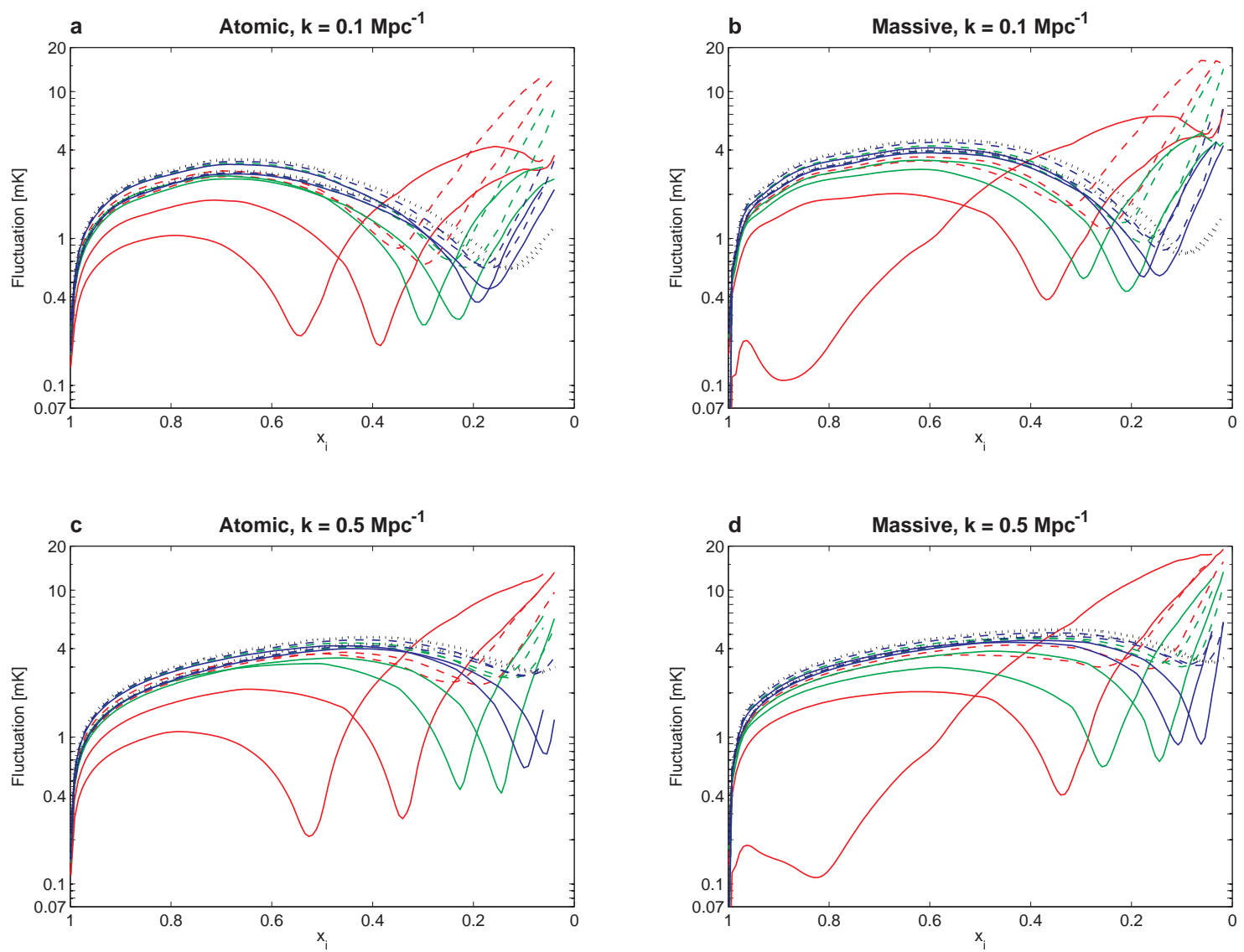

Figure 3: The 21-cm power spectrum. We show the fluctuation level, defined as $\left[k^{3} P(k) /\left(2 \pi^{2}\right)\right]^{1 / 2}$ in terms of the power spectrum $P(k)$ of the $21-\mathrm{cm}$ brightness temperature fluctuations, versus the ionized (mass) fraction of the universe $x_{i}$ (starting on the right from $z=15$ ). We compare the new XRB spectrum ${ }^{9}$ (solid curves) to the previously-adopted soft spectrum (dashed curves), and show the saturated heating case for reference (black dotted curves). We consider our standard $f_{X}=1$ case (green curves), as well as $f_{X}=1 / \sqrt{10}$ (red curves) or $\sqrt{10}$ (blue curves), each with either early or late reionization. We consider wavenumber $k=0.1 \mathrm{Mpc}^{-1}$ (a and $\mathbf{b}$ ) or $k=0.5 \mathrm{Mpc}^{-1}$ (c and $\mathbf{d}$ ), for each of our two cases for galactic halos, atomic cooling (a and c) or massive halos (b and d). Our lower $k$ value roughly tracks large-scale fluctuations (heating early on, and ionized bubbles later), while our higher $k$ value corresponds to a smaller scale (though one that can still be measured accurately with current experiments) and thus tracks more closely the evolution of density fluctuations. To illustrate the effect of the X-ray spectrum on the results, consider the fluctuation level at $k=0.5 \mathrm{Mpc}^{-1}$ at the mid-point of reionization (i.e., $x_{i}=0.5$ ); the parameter space we explore gives a possible range of 3.6-4.9 mK for the old spectrum, while the new spectrum gives a much broader range of 0.3-4.4 mK. Note also that the latter values are typically much lower than the often-assumed limit of saturated heating (which gives a corresponding range of 4.1-5.1 mK). 
Depending on the parameters, the deep minimum (reaching below $1 \mathrm{mK}$ ) may occur at any time during reionization, but is likely to occur before its mid-point. Previously, the fluctuation signal was expected to lie within a narrow, well-defined range, allowing for a straight-forward interpretation of the data in terms of the progress of reionization; now, however, there is a variety of possibilities (Fig. 3), so modeling of data will involve an analysis of the interplay of heating and reionization.

While ongoing experiments hope to reach a sub-mK sensitivity level ${ }^{7.23}$, the best current upper limit ${ }^{24}$ of $52 \mathrm{mK}$ at $k=0.075 \mathrm{Mpc}^{-1}$ at $z=7.7$ is two orders of magnitude away from our predictions. If a sufficient sensitivity level can be achieved, a low minimum in the $21-\mathrm{cm}$ power spectrum during reionization would be a clear signature of late heating due to a hard Xray spectrum. Indeed, a clear observational indication that this feature corresponds to a cosmic milestone is that the minima at all $k>0.5 \mathrm{Mpc}^{-1}$ should occur at essentially the same redshift (namely the true redshift of the heating transition); the minima at lower wavenumbers should be delayed due to rapid evolution in the power spectrum shape (see Extended Data Fig. 2).

Heating by high-energy X-rays would remove the previously expected signal from an early heating transition ${ }^{10,22}$ at $z \sim 15-20$, but would leave in place the similar $z \sim 25$ signal from the Lyman- $\alpha$ coupling transition that is likely detectable with the Square Kilometre Array 25,26 . It could also affect other observations of high-redshift galaxies. For example, since late heating implies weak photoheating feedback, low-mass halos may continue to produce copious stars in each region right up to its local reionization, although internal feedback (arising from supernovae or mini-quasars) could still limit star formation in small halos.

\section{Methods Summary}

Our calculations are performed with a hybrid, semi-numerical method that produces realistic, three-dimensional realizations of the early galaxies along with self-consistent inhomoge- 

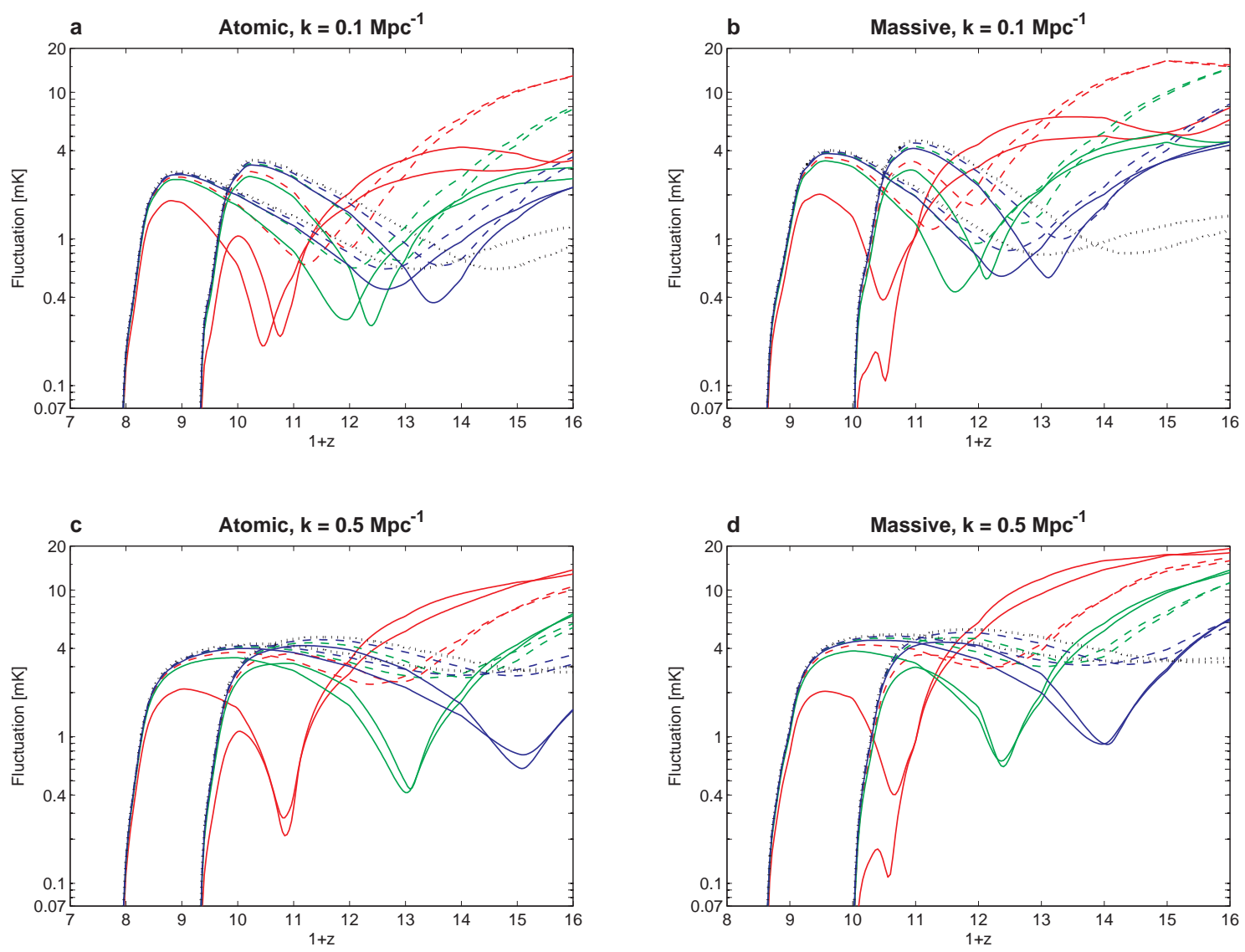

Figure 4: Extended Data Figure 1: The 21-cm power spectrum versus redshift. We show the same data as in Figure 3 (with the same nomenclature), but as a function of $1+z$ where $z$ is the redshift, a direct observable (since the observed wavelength is $21(1+z) \mathrm{cm}$ ). This presentation has the advantage of clearly separating out the early and late reionization cases, while showing that reionization does not affect the redshift of the new minimum (solid curves) at $k=0.5 \mathrm{Mpc}^{-1}$. Indeed, this minimum marks the cosmic heating transition (to within $2 \%$ in redshift in all our model calculations), while the minimum at $k=0.1 \mathrm{Mpc}^{-1}$ is typically delayed due to the evolving power spectrum shape (see Extended Data Fig. 2). We consider wavenumber $k=0.1 \mathrm{Mpc}^{-1}$ (a and b) or $k=0.5 \mathrm{Mpc}^{-1}$ (c and $\mathbf{d}$ ), for each of our two cases for galactic halos, atomic cooling (a and $\mathbf{c}$ ) or massive halos (b and $\mathbf{d}$ ). The shown results (here and in Figure 3) correspond to a total of four different reionization histories. Late reionization with atomic cooling reaches $1 / 4,1 / 2,3 / 4$, and full reionization at $z=10.7,8.7,7.7$, and 7.0 ; the corresponding redshifts for early reionization are 11.8, 10.0, 9.1, and 8.4. Massive halos give a sharper reionization transition, with late reionization advancing through $z=10.3,8.9,8.3$, and 7.7, while early reionization corresponds to $z=11.4,10.2,9.7$, and 9.0 . 

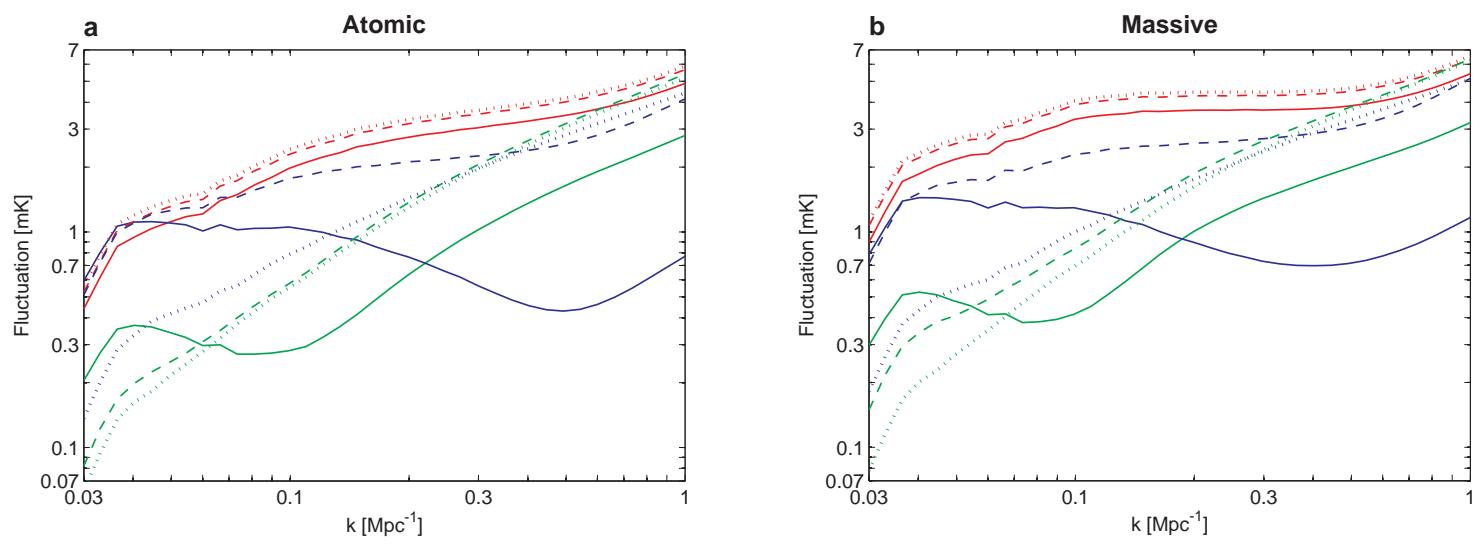

Figure 5: Extended Data Figure 2: Full 21-cm power spectra. We show examples of full power spectra corresponding to the data shown in Fig. 3 and Extended Data Fig. 1, for the cases with $f_{X}=1$ and late reionization. We compare the new XRB spectrum ${ }^{9}$ (solid curves) to the previously-adopted soft spectrum (dashed curves), and show the saturated heating case for reference (dotted curves). We consider atomic cooling (a) or massive halos (b). In order of increasing cosmic age, we consider three key moments (which fall at different redshifts for the various cases, based on Extended Data Fig. 1): the minimum fluctuation at $k=0.5 \mathrm{Mpc}^{-1}$ (blue curves), the minimum at $k=0.1 \mathrm{Mpc}^{-1}$ (green curves), and the mid-point of reionization (red curves). For the new spectrum, strong evolution is predicted in the power spectrum shape, as large-scale fluctuations from X-ray heating dominate (up to $k \sim 0.5 \mathrm{Mpc}^{-1}$ ) at the heating transition (blue solid curves) but then rapidly decline so that density fluctuations come to dominate (at $k>0.1 \mathrm{Mpc}^{-1}$ at the time shown in green solid curves), with an eventual large-scale boost by the ionized bubbles (red solid curves). 
neous distributions of the important radiation backgrounds. This method improves upon our

own and others' previous work $\frac{12.19,22.26}{2}$. In particular, we have added to our previous code a calculation of reionization (by stellar ultraviolet photons) and of partial ionization by X-rays. Our approach is similar to the 21CMFAST code $\frac{12}{2}$ except that in the heating code we calculate the X-ray optical depth (as a function of position and of frequency) much more accurately.

\section{References}

1. Barkana, R., Loeb, A. Unusually Large Fluctuations in the Statistics of Galaxy Formation at High Redshift. Astrophys. J. 609, 474-481 (2004)

2. Furlanetto, S. R., Zaldarriaga, M., Hernquist, L. The Growth of H II Regions During Reionization. Astrophys. J. 613, 1-15 (2004)

3. Mellema, G., Iliev, I. T., Pen, U.-L., Shapiro, P. R. Simulating cosmic reionization at large scales - II. The 21-cm emission features and statistical signals. Mon. Not. R. Astron. Soc. 372, 679-692 (2006)

4. Zahn, O., Lidz, A., McQuinn, M., Dutta, S., Hernquist, L., Zaldarriaga, M., Furlanetto, S. R. Simulations and Analytic Calculations of Bubble Growth during Hydrogen Reionization. Astrophys. J. 654, 12-26 (2007)

5. Madau, P., Meiksin, A., Rees, M. J. 21 Centimeter Tomography of the Intergalactic Medium at High Redshift. Astrophys. J. 475, 429-444 (1997)

6. Furlanetto, S. R. The global 21-centimeter background from high redshifts. Mon. Not. R. Astron. Soc. 371, 867-878 (2006)

7. Furlanetto, S. R., Oh, S. P., Briggs, F. H. Cosmology at low frequencies: The $21 \mathrm{~cm}$ transition and the high-redshift Universe. Phys. Rep. 433, 181-301 (2006) 
8. Fragos, T., et al. X-Ray Binary Evolution Across Cosmic Time. Astrophys. J. 764, 41 (2013)

9. Fragos, T., Lehmer, B. D., Naoz, S., Zezas. A., Basu-Zych, A. Energy Feedback from X-Ray Binaries in the Early Universe. Astrophys. J. 776, 31 (2013)

10. Pritchard, J. R., Furlanetto, S. 21-cm fluctuations from inhomogeneous X-ray heating before reionization. Mon. Not. R. Astron. Soc. 376, 1680-1694 (2007)

11. Christian, P., Loeb, A. Measuring the X-ray Background in the Reionization Era with First Generation $21 \mathrm{~cm}$ Experiments. J. Cosmo. Astropart. Phys. 09, 014 (2013)

12. Mesinger, A., Furlanetto, S., Cen, R. 21CMFAST: a fast, seminumerical simulation of the high-redshift 21-cm signal. Mon. Not. R. Astron. Soc. 411, 955-972 (2011)

13. Mesinger, A., Ferrara, A., Spiegel, D. S. Signatures of X-rays in the early Universe, Mon. Not. R. Astron. Soc. 431, 621-637 (2013)

14. Bennett, C. L., et al. Nine-Year Wilkinson Microwave Anisotropy Probe (WMAP) Observations: Final Maps and Results. Astrophys. J. Supp. 208, 20 (2013)

15. Ade, P. A. R., et al. Planck 2013 results. XVI. Cosmological parameters. arXiv:1303.5076 (2013)

16. Schroeder, J., Mesinger, A., Haiman, Z. Evidence of Gunn-Peterson damping wings in high-z quasar spectra: strengthening the case for incomplete reionization at $z \sim 6-7$. Mon. Not. R. Astron. Soc. 428, 3058-3071 (2013)

17. Haiman, Z., Rees, M. J., Loeb, A. Destruction of Molecular Hydrogen during Cosmological Reionization. Astrophys. J. 476, 458-463 (1997); erratum - Astrophys. J. 484, 985 (1997) 
18. Ahn, K., Iliev, I. T., Shapiro, P. R., Mellema, G., Koda, J., \& Mao, Y. Detecting the Rise and Fall of the First Stars by Their Impact on Cosmic Reionization. Astrophys. J. 756, L16 (2012)

19. Fialkov, A., Barkana, R., Visbal, E., Tseliakhovich, D., Hirata, C. M. The 21-cm signature of the first stars during the Lyman-Werner feedback era. Mon. Not. R. Astron. Soc. 432, 2909-2916 (2013)

20. Dekel, A., Silk, J. The origin of dwarf galaxies, cold dark matter, and biased galaxy formation. Astrophys. J. 303, 39-55 (1986)

21. Wyithe, J. S. B., Loeb, A. A suppressed contribution of low-mass galaxies to reionization due to supernova feedback. Mon. Not. R. Astron. Soc. 428, 2741-2754 (2013)

22. Visbal, E., Barkana, R., Fialkov, A., Tseliakhovich, D., Hirata, C. M. The signature of the first stars in atomic hydrogen at redshift 20. Nature 487, 70 (2012)

23. McQuinn, M., Zahn, O., Zaldarriaga, M., Hernquist, L., Furlanetto, S. R. Cosmological Parameter Estimation using $21 \mathrm{~cm}$ Radiation from the Epoch of Reionization. Astrophys. J. 653, 815-834 (2006)

24. Parsons, A. R., et al. New limits on $21 \mathrm{~cm}$ EoR from PAPER-32 consistent with an X-ray heated IGM at $z=7.7$. $\operatorname{arXiv:1304.4991(2013)}$

25. Barkana, R., Loeb, A. Detecting the Earliest Galaxies through Two New Sources of 21 Centimeter Fluctuations. Astrophys. J. 626, 1-11 (2005)

26. Fialkov, A., Barkana, R., Pinhas, A., Visbal, E. Complete history of the observable 21-cm signal from the first stars during the pre-reionization era. Mon. Not. R. Astron. Soc. 437, L36-L40 (2014) 
27. McClintock, J. E., Remillard, R. A. Black hole binaries. In: Compact stellar X-ray sources. Edited by Walter Lewin \& Michiel van der Klis, Cambridge Astrophysics Series, 157-213 (2006)

28. Tamura, M., Kubota, A., Yamada, S., et al. The Truncated Disk from Suzaku Data of GX 339-4 in the Extreme Very High State. Astrophys. J. 753, 65 (2012)

29. Naoz, S., Noter, S., Barkana, R. The first stars in the Universe. Mon. Not. R. Astron. Soc. 373, L98-L102 (2006)

30. Fialkov, A., Barkana, R., Tseliakhovich, D., Hirata, C. Impact of the relative motion between dark matter and baryons on the first stars: semi-analytical modelling. Mon. Not. R. Astron. Soc. 424, 1335-1345 (2012)

Acknowledgments We are grateful to Smadar Naoz for drawing our attention to the work of Tassos Fragos, who kindly provided us with detailed model spectra of X-ray binaries, which helped motivate this study. This work was supported by Israel Science Foundation grant 823/09, and by the LabEx ENS-ICFP: ANR-10-LABX-0010/ANR-10-IDEX-0001-02 PSL*.

Author Contributions RB initiated the project, AF developed and ran the simulations and made the figures by substantially extending a code originally developed by EV working with $\mathrm{RB}$. The text was written by RB and edited by the other authors.

Author Information Reprints and permissions information is available at www.nature.com/reprints. Correspondence and requests for materials should be addressed to A.F. (anastasia.fialkov@gmail.com) or R.B. (barkana@wise.tau.ac.il). 


\section{Methods}

\section{Hybrid simulation code}

For this work we extended our independent code that we had previously developed $22,19.26$. This code implements a hybrid, semi-numerical method to produce instances of the expected

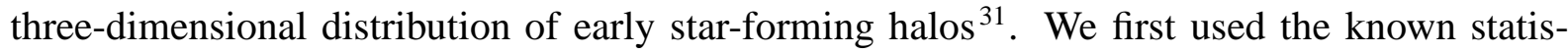
tical properties of the initial density perturbations to generate a realistic sample universe on large, linear scales. Specifically, we assumed Gaussian initial conditions and adopted the initial power spectrum corresponding to the currently best-measured cosmological parameters from the Planck satellite $\stackrel{15}{ }$. In a cubic volume consisting of $128^{3}$ cells (each 3 comoving Mpc on a side), we generated as in our previous work a random realization of the initial overdensity (with periodic boundary conditions). Our code also fully includes the effect of the spatiallyvarying relative baryon-dark matter velocity $\frac{32.22}{2}$ as well as inhomogeneous Lyman-Werner feedback $\frac{17,18,19}{}$, but these play a very minor role in this paper, since we focused here on relatively late times at which star formation is dominated by relatively high-mass halos.

Given the large-scale density distribution, we then computed the gas fraction in star-forming halos in each cell as a function of time, as in our previous papers. Specifically, this gas has density

$$
\rho_{\text {gas }}=\int_{M_{\text {cool }}}^{\infty} \frac{d n}{d M} M_{\text {gas }}(M) d M,
$$

where $d n / d M$ is the comoving abundance of halos of mass $M$ (i.e., $n$ is the comoving number density), $M_{\text {gas }}(M)$ is the gas mass inside a halo of total mass $M$, and $M_{\text {cool }}$ is the minimum halo mass in which the gas can cool efficiently and form stars. The stellar density equals $\rho_{\text {gas }}$ multiplied by the star-formation efficiency.

We then used this information to determine the X-ray heating rate in each cell as follows. At each redshift, we smoothed the stellar density field in shells around each cell, by filtering 
it (using fast Fourier transforms) with two position-space top-hat filters of different radii and taking the difference. We assumed the flux of X-ray photons emitted from each shell to be proportional to the star formation rate (eq.11), which is in turn proportional to the time derivative of $\rho_{\text {gas }}$. We then computed the heating by integrating over all the shells seen by each cell, as in the $21 \mathrm{CMFAST} \operatorname{code}^{12}$. In this integral, the radiative contribution of each cell to a given central cell was computed at the time-delayed redshift seen by the central cell, using a pre-computed interpolation grid of star formation versus overdensity and redshift. We varied the number and thickness of shells to check for convergence. We used photoionization cross sections and energy deposition fractions from atomic physics calculations 33,34 .

Given the X-ray heating rate versus redshift at each cell, we integrated as in 21CMFAST to get the gas temperature as a function of time. We interpolated the heating rate between the redshifts where it was explicitly computed, and varied the number of redshifts to ensure convergence. Our code also fully includes the inhomogeneous coupling between the $21-\mathrm{cm}$ spin temperature and the gas temperature due to Lyman- $\alpha$ radiation ${ }^{26}$, but in this paper we focused on late times when the Lyman- $\alpha$ coupling has nearly saturated, and thus the $21-\mathrm{cm}$ spin temperature equals the kinetic gas temperature to high accuracy. Note, however, that the incomplete saturation has a small effect at the high-redshift $(z \sim 15)$ end of Fig. 3 and Extended Data Figs. 1 and 2; also, it is the main driver of the high-redshift portion of the global signal shown in Fig. 2.

The $21-\mathrm{cm}$ brightness temperature (relative to the $\mathrm{CMB}$ temperature $T_{\mathrm{CMB}}$ ) equals $\underline{\underline{5}}$

$$
T_{21}=29(1+\delta) x_{H I}\left(1-\frac{T_{\mathrm{CMB}}}{T_{\mathrm{S}}}\right) \sqrt{\frac{1+z}{10}} \mathrm{mK}
$$

where $T_{\mathrm{S}}$ is the spin temperature of hydrogen, $\delta$ the overdensity, and $x_{H I}$ the neutral fraction of hydrogen. In our code we also included a small modification of eq. 3 at low gas temperatures 35,36 . We calculated reionization maps as in $21 \mathrm{CMFAST}^{12}$, accounting for the effect of 
large-scale galaxy fluctuations $\frac{1}{\underline{\underline{1}}}$ by setting each cell to be fully reionized if some sphere around it contains enough ionizing photons to self-reionize ${ }^{2}$; we considered spheres up to 70 comoving Mpc, roughly the maximum size of ionized bubbles at the end of reionization $\frac{37}{}$. We took an ionization threshold corresponding to 3 ionizations of each atom due to recombinations. This number can be lowered without changing our results if the escape fraction of ionizing photons is correspondingly lowered as well. In any case, as mentioned in the main text, we set the effective ionization efficiency (in our early and late reionization cases) according to empirical limits on reionization.

As also noted in the main text, we considered two possibilities for the minimum mass of dark matter halos that host galaxies. Specifically, we considered a minimum mass set by the need for efficient atomic cooling (corresponding to a minimum halo circular velocity of $16.5 \mathrm{~km} / \mathrm{s}$ ), or a "massive halo" case with a minimum mass higher by a factor of 9.5 (corresponding to a minimum circular velocity of $35 \mathrm{~km} / \mathrm{s}$ ). We adopted a star formation efficiency of $f_{*}=0.05$ for our atomic cooling case and $f_{*}=0.15$ for massive halos, so that in our model both cases require an escape fraction of ionizing photons of $\sim 20 \%$ for our late reionization case and $\sim 40 \%$ for early reionization. Taking lower $f_{*}$ would push the escape fraction towards unrealistically high values, while higher $f_{*}$ would strongly contradict recent numerical simulations that suggest relatively low values for such halos $\underline{38,39}$.

Other than reionization, another addition to our code (compared to our previous publications) was partial ionization by the same $\mathrm{X}$-rays that heat the gas. These $\mathrm{X}$-rays make a negligible contribution to reionization compared to stellar ultraviolet photons, but their slight ionization of gas that is still mostly neutral affects heating through the ionization dependence of various cross-sections $\frac{33}{}$. In the calculation of heating and ionization by X-rays, our code accurately follows the inhomogeneous X-ray optical depth (though with the approximation of a cosmic mean density) between sources and absorbers. In particular, it includes two substantial advantages 
over $21 \mathrm{CMFAST} \stackrel{12}{ }$. First, when the universe begins to reionize, we do not use the cosmic mean ionization fraction for the optical depth, but rather we use the local, spherically-averaged value (including time-retardation) in the region between each spherical shell of X-ray sources and the central, absorbing pixel. This means that our hybrid simulation correctly heats the gas immediately surrounding $\mathrm{H}$ II regions especially strongly, due both to the presence of an overdensity of sources nearby (within the ionized regions) and the low optical depth to these sources (because most of the intervening gas is ionized). And second, we do not use the two-step approximation of optical depth in 21CMFAST, whereby the effective optical depth is either 0 or $\infty$, but instead we account for the correct (spherically averaged) optical depth seen by photons of each frequency from each spherical shell.

\section{Rough estimate of X-ray heating}

We can understand the critical parameters involved in cosmic heating with a simple estimate. The amount of energy inserted into the gas by X-ray sources is determined by the collapse fraction $f_{\text {coll }}$ (i.e., the fraction of the cosmic gas that makes it into star-forming dark matter halos), the star-formation efficiency $f_{*}$, the fraction $f_{\text {abs }}$ of the X-ray energy that is absorbed by the gas (after losses due to redshift effects), and the fraction $f_{\text {heat }}$ of the absorbed X-ray energy that goes into heating. A simple estimate of the effect of X-ray heating is given by equating the X-ray heating energy to the thermal energy of the gas, yielding the resulting gas temperature (neglecting adiabatic and Compton cooling or heating). At redshift $z$, the ratio of this gas temperature from $\mathrm{X}$-ray heating to the $\mathrm{CMB}$ temperature is

$$
\frac{T_{X}}{T_{\mathrm{CMB}}} \approx 9 f_{X} \frac{f_{\text {coll }}}{0.01} \frac{f_{*}}{0.05} \frac{f_{\text {abs }}}{0.8} \frac{f_{\text {heat }}}{0.12} \frac{10}{1+z},
$$

where we have assumed the $L_{X} /$ SFR ratio from eq. 1. Thus, it is generally assumed that reionization occurs when $T_{\text {gas }} \gg T_{\mathrm{CMB}}$, i.e., much later than the heating transition (when the mean gas temperature equals that of the $\mathrm{CMB}$ ), in the regime of saturated heating when the 
21-cm emission is independent of the gas temperature. As explained in the main text, the new XRB spectrum that we adopt $\stackrel{9}{-}$ reduces $f_{\text {abs }}$ by a factor of 5 and changes this conclusion.

We note that this estimate assumes our standard ratio in eq. 1, which includes an order of magnitude increase in this ratio at the low metallicity expected for high redshift galaxies compared to the local ratio at solar metallicity. This increase is predicted by the evolutionary models and suggested (though not yet solidly confirmed) by observations $\frac{9.40 .41 .42}{\text {; not including it would }}$ only strengthen our case for a late heating. We also note that our standard ratio includes intrinsic (i.e., interstellar) absorption, assuming that the strength of this absorption at high redshift is similar to that at low redshift $\stackrel{9}{\text {. }}$

A similar value for the $L_{X}$ /SFR ratio has been previously claimed for local galaxies ${ }^{6}$; this high value was reached by extrapolating hard (2-10 keV) X-ray observations down to $0.2 \mathrm{keV}$ according to the previously adopted soft spectrum (Fig. 1), resulting in an overestimate by a factor of $\sim 3$. This leaves a further factor of 3 discrepancy (which may mostly be due to the difference between the SFR of high-mass stars as sometimes used ${ }^{43}$ compared to the total SFR). We also note that we have used in eq. 4 the rather low value of $f_{\text {heat }}$ appropriate for gas $\frac{33}{3}$ with the residual ionized fraction of $\sim 2 \times 10^{-4}$ expected before $\mathrm{X}$-rays begin to raise this value.

\section{Other heating sources}

In this paper we have focused on X-ray binaries as the most natural high-redshift heating source, since they are a direct consequence of the star formation believed responsible for reionizing the universe, and high-redshift XRB populations should be generally similar to those observed in the local universe (except for some differences due to metallicity). We note that XRBs from the high redshifts considered here make only a small contribution to the observed X-ray background 9 .

Based on low-redshift observations, other potential X-ray sources appear sub-dominant compared to XRBs. One such source is thermal emission from hot gas in galaxies. The ratio 
of its X-ray luminosity to the SFR in local galaxies ${ }^{44}$ is $\sim 10^{39}$ (including intrinsic absorption). This is well below even the local ratio for XRBs, so it should make only a minor contribution at high redshift (compared with eq. 1). Some theoretical arguments suggest that X-rays produced via Compton emission from relativistic electrons could be important at high redshift ${ }^{45}$, but the expected spectrum (flat from $\sim 100 \mathrm{eV}$ to $\sim 100 \mathrm{GeV}$ ) would deliver most of its energy above $1 \mathrm{keV}$ and thus produce similar 21-cm signatures as our assumed XRB spectrum.

Another possible X-ray source is the population of bright quasars. Although quasars are believed to dominate the X-ray background at low redshift ${ }^{46}$, their rapid decline beyond $z \sim 3$ suggests that their total X-ray luminosity (including an extrapolation of their observed luminosity function) is sub-dominant compared to XRBs during and prior to reionization ${ }^{9}$.

More promising perhaps is the possibility of a population of mini-quasars, i.e., central black holes in early star-forming halos. This must be considered speculative, since the early halos are so small compared to galactic halos in the present universe that the corresponding blackhole masses should fall in a very different range from observed quasars, specifically within the intermediate black-hole range $\left(10^{2}-10^{4} M_{\odot}\right)$ that local observations have probed only to a limited extent ${ }^{47}$. Thus, the properties of these mini-quasars are highly uncertain, and various assumptions can allow them to produce either early or late heating 48,49 .

We can use local observations to try to estimate the possible importance of mini-quasars. An internal feedback model that is consistent with observations of local black-hole masses as well as high-redshift quasar luminosity functions suggests the following relation between the black-hole $(\mathrm{BH})$ mass and the mass of its host dark matter halo $\underline{50}$ :

$$
M_{\mathrm{BH}}=1.5 \times 10^{-6} M_{\text {halo }}\left(\frac{M_{\text {halo }}}{10^{8} M_{\odot}}\right)^{2 / 3}\left(\frac{1+z}{10}\right)^{5 / 2} .
$$

Assuming that mini-quasars shine at the Eddington luminosity, and that this luminosity comes 
out in X-rays, gives

$$
L_{X}=1.5 \times 10^{40} \frac{\mathrm{erg}}{\mathrm{s}}\left(\frac{M_{\mathrm{halo}}}{10^{8} M_{\odot}}\right)^{5 / 3}\left(\frac{1+z}{10}\right)^{5 / 2} .
$$

Next, we assume that each mini-quasar shines at this luminosity for a lifetime equal to the dynamical time of its galactic disk and that the time between active periods is given by the merger timescale ${ }^{50}$. Assuming that this merger timescale is roughly equal to the star formation timescale (which is defined as the ratio between the total mass in stars to the SFR), we find the ratio of the average X-ray luminosity of the central mini-quasar to that of XRBs in the same halo to be:

$$
\frac{\text { Mini }- \text { quasar }}{\text { XRBs }}=0.2\left(\frac{f_{X} f_{*}}{0.05}\right)^{-1}\left(\frac{M_{\text {halo }}}{10^{8} M_{\odot}}\right)^{2 / 3}\left(\frac{1+z}{10}\right),
$$

where we assumed that on average $1 / 4$ of the mini-quasar X-rays in the relevant wavelengths make it past interstellar absorption, similar to observed quasars $\frac{51}{}$ and XRBs $\frac{9.44}{4}$. Thus, this observationally-based estimate indicates a small mini-quasar contribution for our atomic cooling case as well as for massive halos (in the latter case, the increased ratio from the larger typical halo mass is mostly canceled out by the increased star-formation efficiency needed in this case).

We note that a shallower relation between $M_{\mathrm{BH}}$ and the halo velocity dispersion than that assumed in equation 5 would increase the ratio in equation 7 by about an order of magnitude $\frac{52}{}$; however, current data strongly favors a steep relation ${ }^{\underline{53}}$. We also note that standard models of accretion disks $\underline{54}$ around black holes predict that the X-ray spectrum of mini-quasars $\underline{48}$ should peak at $1-5 \mathrm{keV}$, making it a hard spectrum that is much closer to the XRB spectrum we have adopted in this paper than to the soft spectrum usually assumed in previous calculations of X-ray heating. Thus, an unusually large contribution from mini-quasars could produce a somewhat earlier heating transition but it would most likely still be marked by a clear minimum in the 21-cm fluctuations.

Regardless of the source of X-rays, an important parameter is the degree of absorption in 
high-redshift halos compared to locally observed galaxies. If we assume that the gas density in high-redshift halos increases proportionally with the cosmic mean density, then the column density through gas (within a galaxy or a halo) is proportional to $(1+z)^{2} M_{\text {halo }}^{1 / 3}$. This simple relation suggests that absorption of X-rays should increase at high redshift, since the redshift dependence should have a stronger effect than the decrease of the typical halo mass. However, complex astrophysics could substantially affect this conclusion, since the lower binding energy of the gas in low-mass halos could make it easier to clear out more of the blockading gas. In this work we have assumed that the strength of the intrinsic absorption of X-rays from XRBs at high redshift is the same as that observed at low redshift.

There has also been some speculation about heating sources other than X-ray radiation, but none have been shown to be important. In particular, heating due to Lyman- $\alpha$ photons is very inefficient ${ }^{55.56 .49}$, even compared to the reduced X-ray heating that we find, and shocks can likely heat only a small fraction of the cosmic gas prior to reionization $\underline{57}$.

\section{References}

31. Barkana, R., Loeb, A. In the Beginning: The First Sources of Light and the Reionization of the Universe. Phys. Rep. 349, 125-238 (2001)

32. Tseliakhovich, D., Hirata, C. Relative velocity of dark matter and baryonic fluids and the formation of the first structures. Phys. Rev. D 82, 083520 (2010)

33. Furlanetto, S. R., Stoever, S. J. Secondary ionization and heating by fast electrons. Mon. Not. R. Astron. Soc. 404, 1869 (2010)

34. Verner, D. A., Ferland, G. J., Korista, K. T., Yakovlev, D. G. Atomic Data for Astrophysics. II. New Analytic FITS for Photoionization Cross Sections of Atoms and Ions. Astrophys. J. 465, 487 (1996) 
35. Hirata, C. M. Wouthuysen-Field coupling strength and application to high-redshift 21-cm radiation. Mon. Not. R. Astron. Soc. 367, 259 (2006)

36. Chuzhoy, L., Shapiro, P. R. Ultraviolet Pumping of Hyperfine Transitions in the Light Elements, with Application to $21 \mathrm{~cm}$ Hydrogen and $92 \mathrm{~cm}$ Deuterium Lines from the Early Universe. Astrophys. J. 651, 1 (2006)

37. Wyithe, J. S. B., Loeb, A. A characteristic size of $\sim 10 \mathrm{Mpc}$ for the ionized bubbles at the end of cosmic reionization. Nature 432, 194 (2004)

38. Safranek-Shrader, C., Milosavljevic, M., Bromm, V. Star Formation in the First Galaxies - II: Clustered Star Formation and the Influence of Metal Line Cooling. arXiv1307.1982 (2013)

39. Wise, J. H., Abel T., Turk, M. J., Norman, M. L., Smith, B. D. The birth of a galaxy - II. The role of radiation pressure. Mon. Not. R. Astron. Soc. 427, 311-326 (2012)

40. Mirabel, I. F., Dijkstra, M., Laurent, P., Loeb, A., \& Pritchard, J. R. Stellar black holes at the dawn of the universe. Astron. \& Astrophys. 528, A149 (2011)

41. Basu-Zych, A. R., et al. The X-Ray Star Formation Story as Told by Lyman Break Galaxies in the 4 Ms CDF-S. Astrophys. J. 762, 45 (2013)

42. Basu-Zych, A. R., et al. Evidence for Elevated X-Ray Emission in Local Lyman Break Galaxy Analogs, Astrophys. J. 774, 152 (2013)

43. Gilfanov, M., Grimm, H.-J., Sunyaev, R. $L_{X}$-SFR relation in star-forming galaxies. Mon. Not. R. Astron. Soc. 347, L57 (2004)

44. Mineo, S., Gilfanov, M., Sunyaev, R. X-ray emission from star-forming galaxies - II. Hot interstellar medium. Mon. Not. R. Astron. Soc. 426, 1870 (2012) 
45. Oh, S. P. Reionization by Hard Photons. I. X-Rays from the First Star Clusters. Astrophys. J. 553, $499(2001)$

46. Vasudevan, R. V., Mushotzky, R. F., Gandhi, P. Can We Reproduce the X-Ray Background Spectral Shape Using Local Active Galactic Nuclei? Astrophys. J. Lett. 770, L37 (2013)

47. Lützgendorf, N., Kissler-Patig, M., Neumayer, N., et al. $M_{\star}-\sigma$ relation for intermediatemass black holes in globular clusters. Astron. \& Astrophys. 555, A26 (2013)

48. Tanaka, T., Perna, R., Haiman, Z. X-ray emission from high-redshift miniquasars: selfregulating the population of massive black holes through global warming. Mon. Not. R. Astron. Soc. 425, 2974 (2012)

49. Ciardi, B., Salvaterra, R., Di Matteo, T. Ly $\alpha$ versus X-ray heating in the high-z intergalactic medium. Mon. Not. R. Astron. Soc. 401, 2635 (2010)

50. Wyithe, J. S. B., Loeb, A. Self-regulated Growth of Supermassive Black Holes in Galaxies as the Origin of the Optical and X-Ray Luminosity Functions of Quasars. Astrophys. J. 595, $614(2003)$

51. Sazonov, S. Y., Ostriker, J. P., Sunyaev, R. A. Quasars: the characteristic spectrum and the induced radiative heating. Mon. Not. R. Astron. Soc. 347, 144 (2004)

52. Volonteri, M., Gnedin, N. Y. Relative role of stars and quasars in cosmic reionization. Astrophys. J. 703, 2113-2117 (2009)

53. McConnell, N. J., Ma, C.-P. Revisiting the scaling relations of black hole masses and host galaxy properties. Astrophys. J. 764, 184 (2013)

54. Shakura, N. I., Sunyaev, R. A. Black holes in binary systems. Observational appearance. Astron. \& Astrophys. 24, 337-355 (1973) 
55. Chen, X., Miralda-Escudé, J. The Spin-Kinetic Temperature Coupling and the Heating Rate due to Ly $\alpha$ Scattering before Reionization: Predictions for 21 Centimeter Emission and Absorption Astrophys. J. 602, 1-11 (2004)

56. Chuzhoy, L., \& Shapiro, P. R. Heating and Cooling of the Early Intergalactic Medium by Resonance Photons. Astrophys. J. 655, 843-846 (2007)

57. Furlanetto, S. R., Loeb, A. Large-Scale Structure Shocks at Low and High Redshifts. Astrophys. J. 611, 642-654 (2004) 